\title{
Glutaminase sustains cell survival via the regulation of glycolysis and glutaminolysis in colorectal cancer
}

\author{
ZHOU SONG, BO WEI, CANRONG LU, PEIYU LI and LIN CHEN \\ Department of General Surgery, General Hospital of Chinese People's Liberation Army, Beijing 100853, P.R. China
}

Received February 18, 2016; Accepted May 4, 2017

DOI: $10.3892 / 01.2017 .6538$

\begin{abstract}
Cancer cells remodel their metabolic programs towards aerobic glycolysis and elevated glutaminolysis to meet the requirement $s$ of rapid proliferation. Understanding how cells sense and adapt to these changes may provide new targets for therapeutic intervention. Deamination of glutamine to glutamate by glutaminase (GLS1) is an essential step in glutaminolysis. The present study revealed that the loss of GLS1 expression by RNA interference or inhibitor decreased the proliferation and viability of colorectal cancer (CRC) cells. The abrogation of GLS1 function notably inhibited glutaminolysis and aerobic glycolysis, which resulted in the decrease of internal ATP levels and an increase in cell death. In addition, GLS1 expression was significantly elevated in CRC tissues in comparison with adjacent normal tissues $(\mathrm{P}<0.001)$, which is associated with the cell differentiation status and tumor node metastasis stage. In conclusion, the present study defined a key role of GLS1 in coupling glutaminolysis with elevated glucose uptake and consequently the growth of colon cancer cells. Due to the functional importance of GLS1 in regulating cell metabolism, it was proposed that GLS1 may serve as a target for colorectal cancer therapy.
\end{abstract}

\section{Introduction}

Colorectal cancer (CRC) is a commonly diagnosed cancer. It is the third most common fatal malignancy and third leading cause of cancer-associated mortalities in the western world (1). The etiology of CRC is multifactorial. Due to the aggressiveness of CRC and the lack of targeted therapies, it is necessary

Correspondence to: Dr Lin Chen, Department of General Surgery, General Hospital of Chinese People's Liberation Army, 28 Fuxing Road, Beijing 100853, P.R. China

E-mail: chenlinbj@vip.sina.com

Abbreviations: CRC, colorectal cancer; GLS, glutaminase; BPTES, bis-2-(5-phenylacetamido-1,2,4-thiadiazol-2-yl)ethyl sulfide; TNM, tumor-node-metastasis

Key words: glutaminase, colorectal cancer, glucose, glutamine to investigate the key pathways required for cancer development and progression.

Central metabolic pathways differ between normal and cancer cells in their regulation and dynamics. Cancer cells exhibit increased glucose and glutamine metabolism to fuel their bioenergetic and biosynthetic demands $(2,3)$. The increased aerobic glycolysis properties, which show taking up glucose and converting it into lactate even with the availability of oxygen, are termed the Warburg effect $(4,5)$. However, increased glycolysis alone is insufficient to meet the total metabolic demands of proliferating cancer cells. Actively growing cells depend on glutaminolysis, which catabolizes glutamine to generate ATP and maintain the mitochondrial function for metabolism, which is termed glutamine addiction (6). Elevated glutaminolysis has also been considered as an important hallmark of cancer $(2,7)$.

The conversion of glutamine into glutamate, catalyzed by glutaminase (GLS; the first enzyme in glutaminolysis) is a key process for glutamine-dependent anapleurosis and glutathione biosynthesis (8). There are two different subtypes of GLS: GLS1 (kidney-type) and GLS2 (liver-type) $(9,10)$. The elevated expression of GLS1 has been observed in several types of cancer, including colorectal cancer (11), prostate cancer (12) and breast cancer (13). In addition, it has been reported that multiple genetic factors are implicated in the regulation of GLS1 expression and glutamine metabolism. Myc, as a proto-oncogene, stimulates the uptake and catabolism of glutamine (14). The Myc family member, c-Myc, indirectly stimulates GLS1 expression in Burkitt's lymphoma and prostate cancer cells through suppression of microRNA-23a/b (15). In addition, the mammalian target of rapamycin pathway and the extracellular signal-regulated protein kinase pathway were also shown to be involved in tumor growth through the regulation of glutaminolysis $(16,17)$. Due to the important role of GLS1 in cell survival, a number of small-molecule inhibitors targeting glutaminase have been developed, including bis-2-(5-phenylacetamido-1,2,4-thiadiazol-2-yl)ethyl sulfide (BPTES) (18), compound 968 (19) and CB-839 (13). Although the alterations of cell metabolism have been observed on a large scale in cancer cells, less is known regarding how cells respond to nutrient changes and the outcome in coordinating cell growth in CRCs.

In the present study, to improve the understanding of the role of GLS1 in solid colorectal tumors and its clinical significance, GLS1 expression was diminished by RNA interference 
or inhibitor and the present study focused on the role of GLS1 in CRC cells and its potential association with clinical features. It was demonstrated that the abrogation of GLS1 function notably inhibited glutaminolysis and aerobic glycolysis, which resulted in the decrease of internal ATP levels and cell survival. Accordingly, GLS1 expression was significantly elevated in CRC tissues in comparison with adjacent normal ones, which was associated with the cell differentiation status and tumor-node-metastasis (TNM) stage. These observations highlight the critical associations of GLS1, glucose uptake and tumor progression, and indicate that glutaminase inhibitors may provide therapeutic benefit in CRC treatment through the regulation of glycolysis and glutaminolysis. Due to the functional importance of GLS1 in regulating cell metabolism, it was proposed that GLS1 may serve as a target for colorectal cancer therapy.

\section{Materials and methods}

Cell cultures and reagents. Colorectal cancer cell lines HT-29 and HCT116 were purchased from American Type Culture Collection (Manassas, VA, USA). The two cell lines were cultured in McCoy's 5A modified medium (catalog no. 12330031, Gibco; Thermo Fisher Scientific, Inc., Waltham, MA, USA) supplemented with $10 \%$ fetal bovine serum (FBS) in a humidified atmosphere of $5 \% \mathrm{CO}_{2}$ at $37^{\circ} \mathrm{C}$. Nutrient depletion studies were performed using glucose and glutamine-free Dulbecco's modified Eagle's medium (DMEM) (catalog no. A1443001, Gibco; Thermo Fisher Scientific, Inc.). Reconstituted medium for all experiments was supplemented with $10 \%$ FBS, and, when required, glucose (catalog no. A2494001, Gibco; Thermo Fisher Scientific, Inc.) or glutamine (catalog no. 25030081, Gibco; Thermo Fisher Scientific, Inc.) was added to the medium to final concentrations of 25 and $2 \mathrm{mM}$, respectively. BPTES (SML0601) was purchased from Sigma-Aldrich; Merck KGaA (Darmstadt, Germany) and was dissolved in dimethyl sulfoxide at a concentration of 1, 5 or $10 \mu \mathrm{M}$ for use. The Mission short hairpin (sh)RNA lentiviral transduction particles (non-target, catalog no. SHC002V; and GLS1, catalog no. SHCLNV) were purchased from Sigma-Aldrich; Merck KGaA. The targeting sequence of GLS1 shRNA1 was 5'-GCACAGACATGGTTGGTATAT-3' (clone ID: TRCN0000051135), and GLS1 shRNA2 was 5'-GCCCTG AAGCAGTTCGAAATA-3' (clone ID: TRCN0000051136). HT29 or HCT-116 cells were transfected with GLS1 shRNA1 or shRNA2 respectively. HT29 cells with non-target control or GLS1 shRNA2 were injected into nude mice for xenograft assay. The infection of mission shRNA lentiviruses transduction particles and selection of positive colonies were performed according to the supplier's protocol (Sigma-Aldrich; Merck $\mathrm{KGaA}$ ). The silencing efficacy of shRNAs was validated using western blot analysis, as described subsequently.

Cell growth assays. A total of $1 \times 10^{4}$ HT29 or HCT116 cells were seeded onto each well of a 96-well plate with five replicates for each group in DMEM. On the second day, the medium was changed with or without glucose or glutamine for 5 days. The cell numbers or cell viabilities were determined as below. For the MTT assay, $20 \mathrm{ml}$ of MTT solution $(5 \mathrm{mg} / \mathrm{ml})$ was added to each well, and following $4 \mathrm{~h}$ of incubation at $37^{\circ} \mathrm{C}$, the medium was aspirated and dimethyl sulfoxide was added. The optical density values were determined at $570 \mathrm{~nm}$ using a Sunrise microplate reader (Tecan Austria $\mathrm{GmbH}$, Grödig, Austria). To measure viability by direct counting, cells were collected and stained with $0.4 \%$ Trypan Blue (catalog no. T6146, Sigma-Aldrich; Merck KGaA). Cells excluding and taking up dye in the whole slide were counted on a hemocytometer under phase-contrast microscopy (magnification, x20; model IX50; Olympus Corporation, Tokyo, Japan).

Cell death assay. For the Guava ViaCount assay, 5x10 $0^{5}$ HT29 or HCT116 cells were seeded onto each well of 6-well plate in DMEM. On the second day, the medium was changed with or without glucose or glutamine for $48 \mathrm{~h}$. Subsequently, the cells were trypsinized to produce a single cell suspension and the viable cell number in each well was counted using the ViaCount assay (Guava Technologies, Hayward, CA, USA).

Colony formation assay. Cells were seeded onto $60 \mathrm{~mm}$ dishes at a density of 200 cells/dish. The cells were grown for 2 weeks in McCoy's 5A modified medium (catalog no. 12330031, Gibco; Thermo Fisher Scientific, Inc.) supplemented with $10 \% \mathrm{FBS}$ at $37^{\circ} \mathrm{C}$ in a humidified atmosphere containing $5 \%$ $\mathrm{CO}_{2}$. The colonies were subsequently fixed and stained with $0.1 \%$ crystal violet solution. The colonies were calculated as the mean number of cells in 10 randomly selected fields using phase-contrast microscopy (magnification, x20; model IX50; Olympus Corporation, Tokyo, Japan).

In vivo xenograft assay. Mice were housed in a laminar flow caging system (Thoren Caging Systems, Inc., Hazleton, PA, USA), and all food, bedding and water were autoclaved. Male BALB/c nu/nu mice (Beijing Vital River Laboratory Animal Technology Co., Ltd., Beijing, China) were aged between 4 and 6 weeks and weighted 18-20 g. They were injected subcutaneously in the limb with $1 \times 10^{7}$ HT29 or HCT116 cells (three animals per group). Tumor growth was monitored by measuring tumor size using Vernier calipers every week for a 4-week period and calculating tumor volume using a standard formula: Tumor volume $\left(\mathrm{mm}^{3}\right)=$ width $\left(\mathrm{mm}^{2}\right)$ x length $(\mathrm{mm})$ $\mathrm{x} 0.5$. At the end of the experiment, tumor weight was assessed by sacrificing the mice by heart puncture under ether anesthesia as previously described (20), removing and weighing the tumor.

Western blot analysis. Cells were collected and lysed in lysis buffer $(50 \mathrm{mmol} / 1$ Tris, $\mathrm{pH} \mathrm{7.5,} 250 \mathrm{mmol} / 1 \mathrm{NaCl}, 0.1 \%$ SDS, $2 \mathrm{mM}$ dithiothreitol, $0.5 \% \mathrm{NP}-40,1 \mathrm{mmol} / 1$ phenylmethylsulfonyl fluoride and protease inhibitor cocktail). Protein concentrations were measured using the bicinchoninic assay kit from Thermo Fisher Scientific, Inc. A total of $40 \mu \mathrm{g}$ protein/lane was separated using 10\% SDS-PAGE and transferred onto Hybond enhanced chemiluminescence (ECL) nitrocellulose membranes. Following electrophoresis, proteins were transferred to a polyvinylidene difluoride membranes (EMD Millipore, Billerica, MA, USA). The membrane was blocked with $5 \%$ nonfat milk in phosphate-buffered saline for $1 \mathrm{~h}$ at room temperature and subsequently incubated with antibodies against general GLS1 antibody (catalog no. ab93434, Abcam, Cambridge, UK) and $\beta$-actin antibody 
(catalog no. bs-0061R, Beijing Biosynthesis Biotechnology Co., Ltd., Beijing, China) at a dilution of 1:1,000, overnight at $4^{\circ} \mathrm{C}$. The membrane was then incubated with a goat anti-rabbit immunoglobulin $\mathrm{G}$ conjugated with horseradish peroxidase (1:5,000, catalog no. BA1056, Wuhan Boster Biological Technology, Ltd., Wuhan, China) secondary antibody, for $1 \mathrm{~h}$ at room temperature, and detected using ECL (Pierce, Rockford, IL, USA) or the Odyssey Imaging System (Li-Cor Biosciences, Lincoln, NE, USA).

Metabolic analysis. Glutamine and glutamate levels in the medium were analyzed using Nova Flex (NOVA Biomedical, Waltham, MA, USA). Glucose and lactate levels were determined using respective assay kits purchased from BioVision, Inc. (EZCell ${ }^{\mathrm{TM}}$ Direct Glucose Uptake assay kit, catalog no. K924; EZScreen ${ }^{\mathrm{TM}}$ Lactate Colorimetric assay kit, catalog no. K951, respectively). Data were presented as the mean of triplicates and were presented as a proportion of the control group.

ATP analysis assay. ATP Bioluminescence assay kit CLS II (Roche Applied Science, Madison, WI, USA) was used to determine the intracellular ATP levels. Following treatment, cells were lysed and ATP levels were measured according to the manufacturer's protocol.

Tissue sample study. A total of 50 primary human colorectal tumor tissues and adjacent non-tumor tissues were obtained from Xijing Hospital of Digestive Diseases (Xijing, China; May 2012-January 2014), with informed consent obtained from each patient and approval from the Clinical Research Ethics Committee of Xijing Hospital, the Fourth Military Medical University (Xi'an, China). In each case, a diagnosis of primary colorectal cancer was made. All clinical cancerous specimens were collected through surgery or endoscopy. All specimens were histologically diagnosed by the Department of Pathology, Xijing Hospital, Fourth Military Medical University (Xi'an, China). Information on patient age, sex, size of primary tumor, tumor differentiation, tumor classification and node classification were collected and verified using the previously described system (21) and are summarized in Table I. The associated procedures were performed following ethical and legal standards regarding human subjects.

Immunohistochemistry (IHC). For immunohistochemical analysis, endogenous peroxidase activity was blocked using $3 \% \mathrm{H}_{2} \mathrm{O}_{2}$ for $12 \mathrm{~h}$. Non-specific binding was blocked with mice serum (catalog no. SA1020, Wuhan Boster Biological Technology, Ltd) for $2 \mathrm{~h}$ at room temperature. Subsequently, the slides were incubated with GLS1 antibody (dilution 1:200, catalog no. ab93434, Abcam) in PBS at $4^{\circ} \mathrm{C}$ overnight in a humidified container. Biotinylated goat anti-rabbit immunoglobulin G (1:400; Sigma-Aldrich; Merck KGaA) was incubated with the sections for $1 \mathrm{~h}$ at room temperature. Brown color, indicative of peroxidase activity, was developed by incubating with 0.1\% 3,3-diaminobenzidine (Sigma-Aldrich; Merck KGaA) in PBS with $0.05 \% \mathrm{H}_{2} \mathrm{O}_{2}$ for $5 \mathrm{~min}$ at room temperature. The appropriate positive and negative controls were included in each IHC run. Immunohistochemistry was
Table I. Association between glutaminase 1 expression and colorectal cancer characteristics.

\begin{tabular}{lrrrr}
\hline & \multicolumn{4}{c}{$\begin{array}{c}\text { GLS } \\
\text { expression, } \mathrm{n}\end{array}$} \\
\cline { 3 - 3 } Parameter & Number & - & +-+++ & P-value \\
\hline Total & 50 & 16 & 34 & \\
Sex & & & & $0.189^{\mathrm{a}}$ \\
Male & 26 & 8 & 18 & \\
Female & 24 & 8 & 16 & \\
Differential status & & & & $<0.001^{\mathrm{b}}$ \\
Poor & 9 & 1 & 8 & \\
Moderate & 22 & 5 & 17 & \\
Well & 19 & 10 & 9 & \\
TNM stage & & & & $<0.001^{\mathrm{b}}$ \\
I-II & 8 & 6 & 2 & \\
III & 16 & 5 & 11 & \\
IV & 26 & 5 & 21 &
\end{tabular}

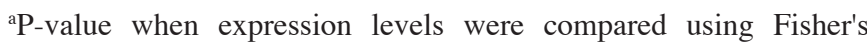
exact test. ${ }^{b} \mathrm{P}-\mathrm{value}$ when expression levels were compared using the Kruskal-Wallis test. GLS1, glutaminase 1; TNM, tumornode-metastasis.

scored as following by 3 investigators: Negative, 1; minimal, 2; moderate, 3, strong, 4; or maximal, 5. Tumors with weak, moderate or strong immunostaining intensity were classified as staining positive $(+)$, whereas tumors with no immunostaining were classified as staining negative (-).

Statistical analysis. Statistical analysis was completed using SPSS software (version 19.0; IBM SPSS, Armonk, NY, USA). All data are presented as the mean \pm standard deviation of triplicate values from 3 separate experiments. $\mathrm{P}<0.05$ was considered to indicate a statistically significant difference. Independent Student's t-test or Student-Newman-Keuls test after one-way analysis of variance was used to compare the continuous variables between the two groups or more than two groups. For clinical sample analysis, Fisher's exact test or Kruskal-Wallis test was used to determine the sex, differentiation status and TNM stage, respectively.

\section{Results}

Glutamine is critical for CRC survival. To determine how glutamine is necessary for the proliferation and survival of colon cancer cells, cell behavior in HT29 and HCT116 cells under different nutrient conditions was determined. The two cell lines exhibited proliferation in the presence of glucose and glutamine, whereas the cells did not grow in the absence of glucose and glutamine, indicating that the two nutrients are necessary for colon cancer cell survival (Fig. 1). In the glutamine withdrawal group, the growth and viability of HT29 and HCT116 cells was also significantly decreased. Similar results were also observed with the guava assay. Glutamine 

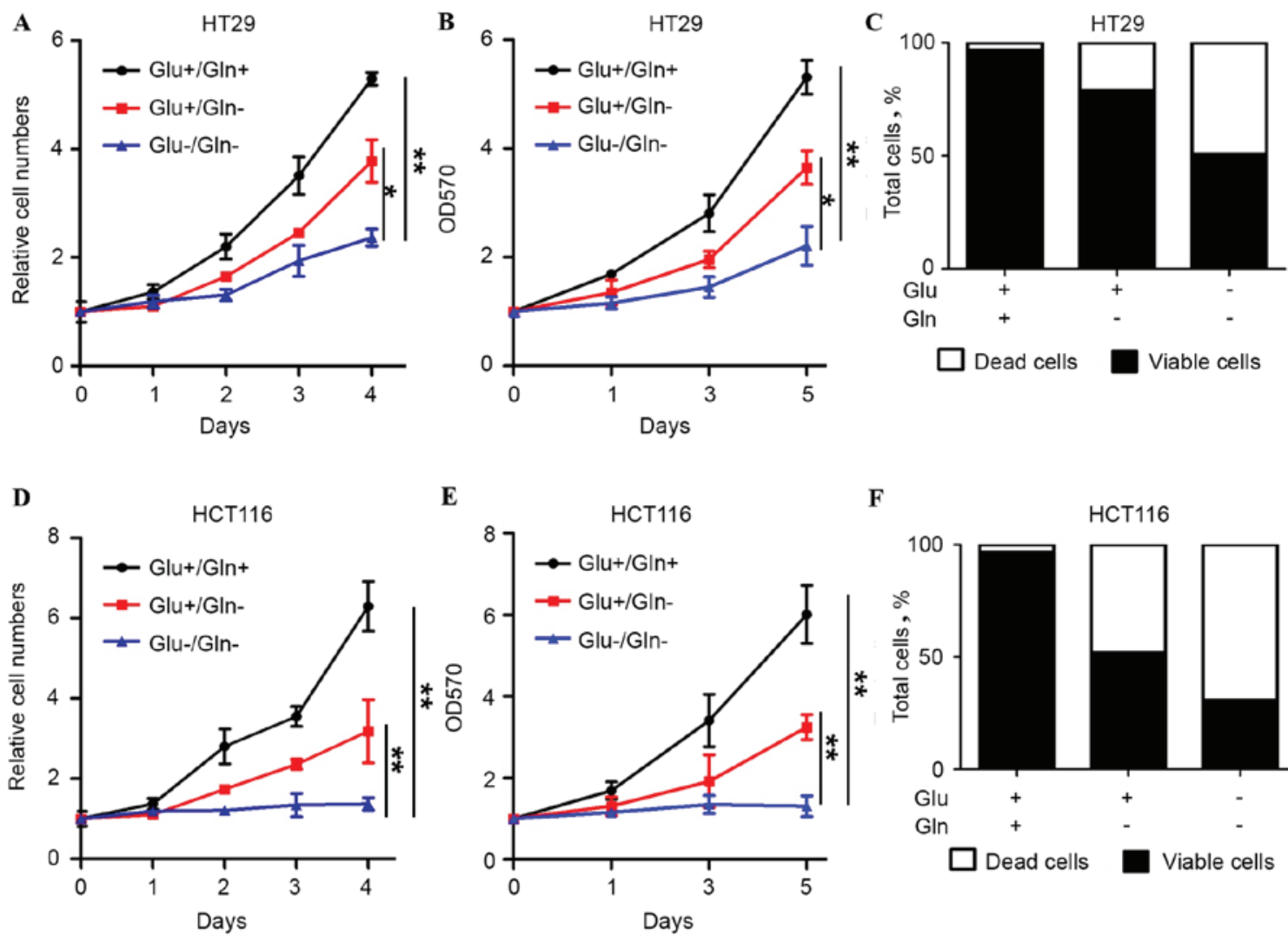

$\mathbf{E}$

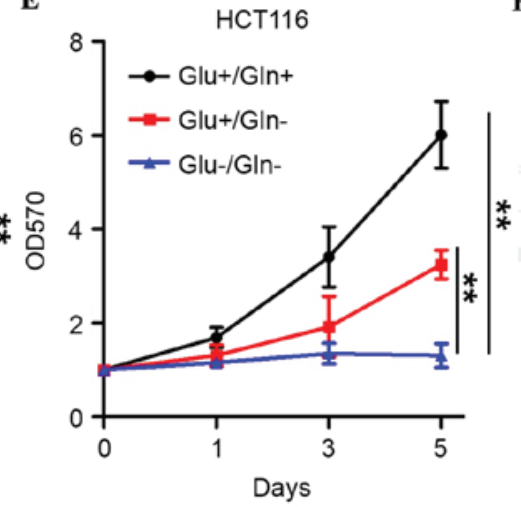

F

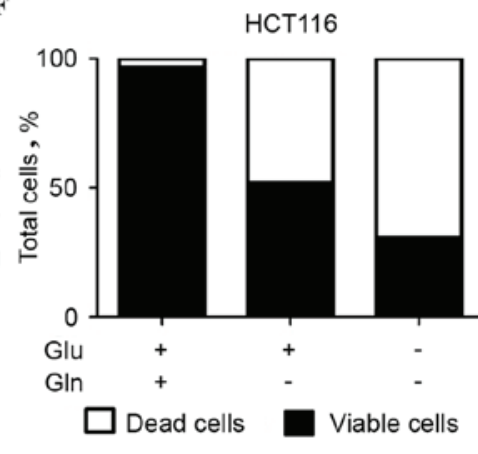

Figure 1. Glutamine is critical for colorectal cancer survival. (A) HT29 and (B) HCT116 cells were grown in medium lacking Gln and/or Glu as indicated over a 4-day time period. Relative cell numbers were determined. (C) The cell viabilities were detected with MTT assay in HT29 and (D) HCT116 cells with indicated treatment conditions. (E) The cell death assays were performed in HT29 and (F) HCT116 cells with indicated treatment conditions. The data are presented as the mean \pm standard deviation from triplicate analyses. ${ }^{*} \mathrm{P}<0.05 ;{ }^{* *} \mathrm{P}<0.01$. Gln, glutamine; Glu, glucose; OD, optical density.

withdrawal caused increased cell death compared with the presence of glucose and glutamine (Fig. 1C and F). Therefore, it was speculated that glutamine, which is an important energy source, is critical for cell growth and the prevention of cell death in colon cancer.

GLS1 sustains CRC cell growth in vitro and in vivo. Due to the key function of GLS1, which converts glutamine into glutamate, it was proposed that GSL1 may be an important factor for the survival of colon cancer. GLS1 was knocked down with two shRNAs, and the two shRNAs showed significant repression of GLS1 expression (Fig. 2A). The cell growth abilities were markedly decreased with GLS1 shRNA transfection (Fig. 2B and C). GLS1 knockdown also inhibited colony formation in HT29 and HCT116 cells (Fig. 2D). To determine whether knockdown of GLS1 can block the cell growth in vivo, HT29 cells transfected with either control or GLS1 shRNA were injected into nude mice and allowed to grow for 4 weeks. The results showed that GLS1 shRNA markedly diminished the capacities of cell growth and tumor formation (Fig. 2E). Furthermore, BPTES (a GLS1 inhibitor) was used as a tool to complement the GLS1 knockdown approach in the present study. Consistent with the aforementioned results, BPTES blocked the growth of colon cancer cells in a dose-dependent manner (Fig. 2F). These results demonstrated that the pleiotropic role of glutamine in tumor growth is dependent on GLS1 activity and glutaminolysis.
GLS1 dictates glycolysis via glutaminolysis. In the majority of proliferating cells, the internal ATP production is predominantly supported by glucose and glutamine. In the present study, it was speculated that the inhibition of colon cancer cell growth with glutamine withdrawal was attributed to the loss of internal ATP production. Therefore, ATP levels were determined with or without glutamine treatment, and it was found that the glutamine withdrawal markedly decreased the internal ATP levels in HT29 and HCT116 cells (Fig. 3A). The reduction of GLS1 by shRNA markedly diminished the cell growth and internal ATP levels (Fig. 3B). Glutamine is converted by GLS1 into glutamate for either glutathione biosynthesis or catabolism by the tricarboxylic acid (TCA) cycle. To evaluate GLS1 oxidative glutamine metabolism, glutamine consumption and glutamate production were analyzed in HT29 and HCT116 cells. As expected, GLS1 depletion significantly suppressed glutamine consumption and subsequent glutamate production (Fig. 3C and D).

A previous study reported that the inhibition of glucose uptake by glutamine was observed in prostate and pancreatic cancer cells $(12,22)$. Therefore, the present study investigated whether this regulatory mechanism is widespread, and is also observed in colon cancer. HT29 and HCT116 cells were transfected with GLS1 shRNA. The GLS1 knockdown markedly decreased cell glucose uptake (Fig. 3E). In addition, the inhibition of GLS1 also blocked lactate production. Thus, in addition to the regulation of glutaminolysis, GLS1 also performs a role 

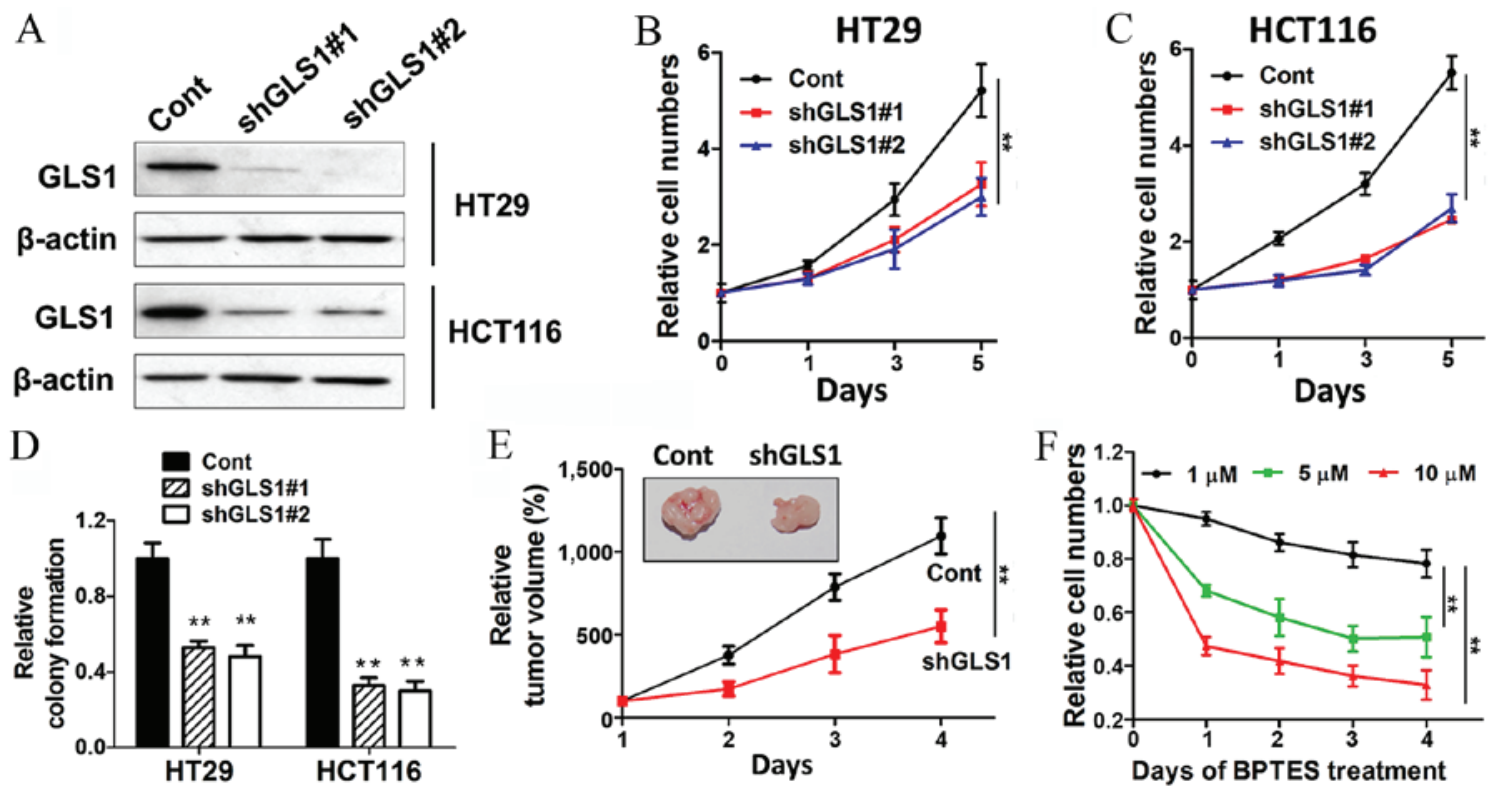

Figure 2. GLS1 sustains colorectal cancer cell growth in vitro and in vivo. (A) Western blot analysis was used to determine the expression of GLS1 in cells with transfected GLS1 shGLS1\#1/shGLS1\#2 or control shRNA. (B) The growth abilities of HT29 and (C) HCT116 cells transfected with shGLS1\#1/shGLS1\#2 or control shRNA. (D) Equal numbers of shGLS1 HT-29/HCT116 cells and control cells were seeded onto $60 \mathrm{~mm}$ dish. After 14 days, the colony formation capacities were determined. (E) Mice were injected subcutaneously with HT29 cells transfected with control or GLS1 shRNA. Tumor volume was detected and calculated. (F) HCT116 cells were grown in the presence of BPTES for the indicated number of days. The cell growth was normalized to its growth in medium without BPTES treatment. Results are presented as the mean \pm standard deviation. ${ }^{*} \mathrm{P}<0.05 ;{ }^{* *} \mathrm{P}<0.01$. GLS1, glutaminase 1 ; shRNA, short hairpin RNA; BPTES, bis-2-(5-phenylacetamido-1,2,4-thiadiazol-2-yl)ethyl sulfide; Cont, control shRNA.

in the regulation of the glucose uptake and glycolysis process. It was proposed that GLS1 controls the coordination between glutamine and glucose, and restricts cell survival as a metabolic checkpoint in colon cancer.

GLS1 expression is increased in CRC tissues and associated with clinicopathological factors. To evaluate the clinical significance of GLS1 in colorectal cancer, the present study examined GLS1 expression in 50 pairs of human colorectal tumors and adjacent normal colon samples with immunohistochemical analysis. As shown in Fig. 4A, the adjacent normal colon tissues did not exhibit significant positive staining for GLS1. By contrast, the expression level of GLS1 was upregulated in the colorectal tumor samples. Statistical analysis revealed that the average fold change of GLS1 expression in colorectal cancer was markedly increased compared with the adjacent normal colon tissues (1.85 vs. 3.53; $\mathrm{P}<0.001$; unpaired Student's t-test; Fig. 4B). To determine the clinical significance of elevated GLS1 expression in patients with CRC, the association between GLS1 protein expression and clinicopathological factors was examined, including sex, age, differentiation status and TNM stage in patients with CRC. The results showed that GLS1 expression was negatively associated with differentiation status, and positively associated with TNM stage (Table I). Therefore, an increase in the percentages and levels of GLS1 positive expression indicated a low differentiation status and an increase in TNM stage in patients with CRC.

\section{Discussion}

Cancer cell metabolism is reprogrammed compared with normal tissue, which results in tumor formation and progression. In addition to elevated aerobic glycolysis, cancer cells exhibit increased dependence on glutamine for growth (23). Since a variety of energy sources enter the TCA cycle in a variety of ways, the restriction of each nutrient may have different effects on cell growth. In rapidly growing cells, glucose-derived citrate is preferentially involved in the synthesis of fatty acids required for cell growth. The importance of glutaminolysis in maintaining the malignant phenotype has also been stressed (15). Thus, glutaminolysis is critical to compensate for the reduced TCA intermediates (3). Targeting glucose metabolism alone may not be sufficient for cancer therapy.

GLS1 performs a critical role in catalyzing glutaminolysis, and connects with a wide variety of distinct biological processes through the conversion of glutamine into glutamate (9). The expression of GLS1 is often upregulated in tumors and rapidly dividing cells (15). Although the underlying mechanism of the elevated GLS1 expression by a number of oncogenes has been reported, the way that GLS1 regulates cell metabolism and growth in CRCs is largely unknown. In the present study, an important role for GLS1 in coupling glutaminolysis of the TCA cycle with elevated aerobic glycolysis in CRCs was defined, which consequently represses the internal ATP levels and cell survival.

The present study firstly demonstrated that HT29 and HCT116 cells have increased sensitivity to the deprivation of glutamine compared with the addition of glucose and glutamine, indicating the glutamine addiction phenotype, which may be considered as a target of interest for therapy. Recently, a study reported that, regarding the heterogeneous genetic and metabolic baseline between HCT116 cells and HT29 cells, HCT116, in contrast to HT29, depends on glutamine 

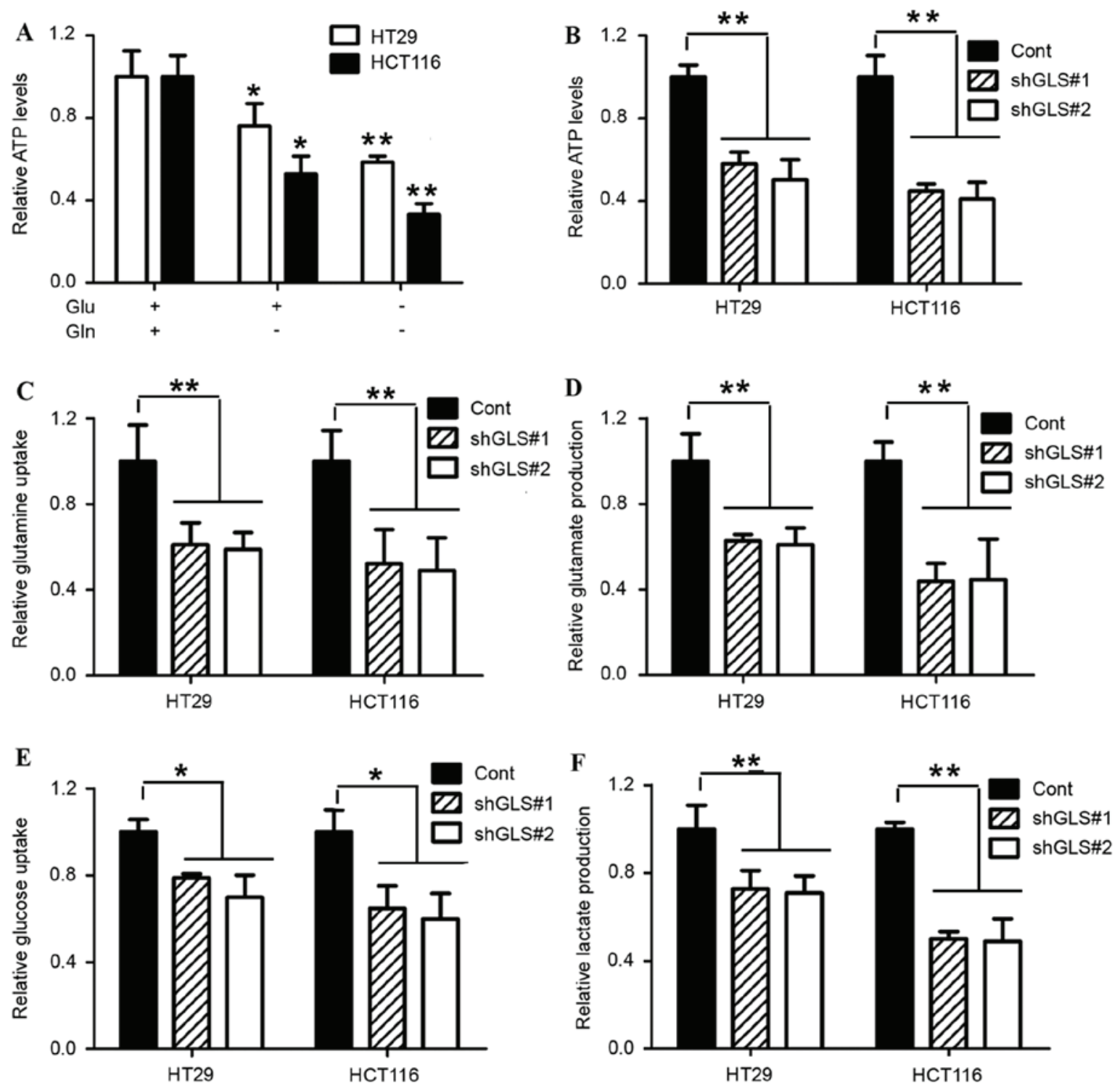

Figure 3. GLS1 dictates glycolysis via glutaminolysis. (A) HT29 and HCT116 cells were cultured in normal medium or medium without glucose or glutamine for $48 \mathrm{~h}$ and harvested for ATP assay. The results exhibited were relative ATP levels per $\mu \mathrm{g}$ of total protein, normalized to the control normal medium group. HT29 and HCT116 cells were transfected with shGLS1\#1/shGLS1\#2 or control shRNA. The (B) internal ATP levels, (C) glutamine uptake, (D) glutamate production, (E) glucose uptake and (F) lactate production were determined relatively. Results are presented as the mean \pm standard deviation. "P $<0.05$; ${ }^{* *} \mathrm{P}<0.01$. GLS1, glutaminase 1; shRNA, short hairpin RNA; Cont, control shRNA.

A

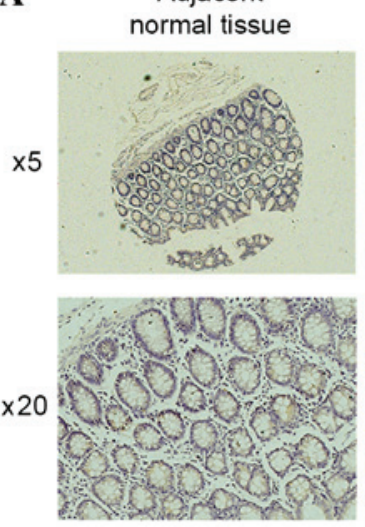

Cancer

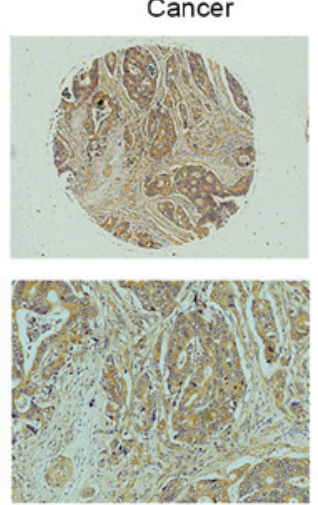

B

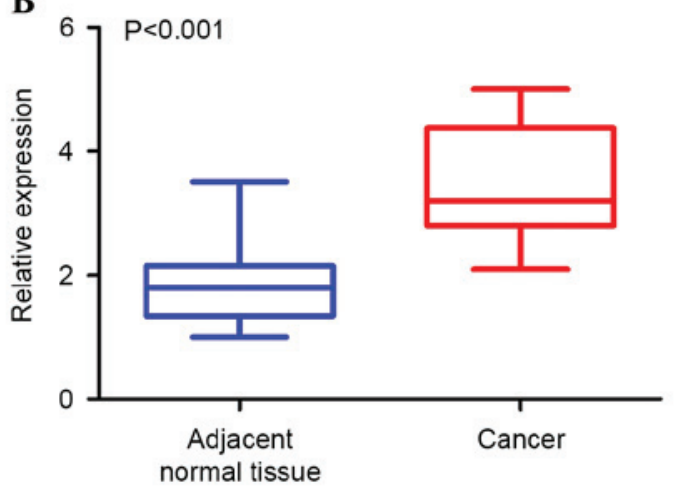

Figure 4. GLS1 expression was increased in colorectal cancer tissues and associated with clinicopathological factors. (A) Immunohistochemical staining of GLS1 in human colorectal tumors and adjacent normal colon samples. Original magnification, x5 or x20. (B) Relative expression level of GLS1 in human tissues. Student's t-test was applied for statistical analysis. GLS1, glutaminase 1. 
metabolism, as compound 968 exhibited increased toxicity in this cell line (24). Accordingly, HCT116 was also observed to be more sensitive to glutamine withdrawal compared with HT29 cells, although the two cell lines require glutamine for survival. In addition, GLS1 was knocked down with shRNA in the two cell lines. The repression of GLS1 decreased cell growth and colony formation in vitro and inhibited the xenograft formation in nude mice. Therefore, glutamine and GLS1 are critical for CRC cell growth and survival.

The coordination of glucose and glutamine as abundant nutrients is required for cell growth, which feeds into multiple pathways. Several studies have provided evidence that glutamine dependent anapleurosis functions upstream of glucose uptake and utilization, and glutaminolysis is required for the uptake of glucose $(12,22)$. It was also observed that the repression of GLS1 decreased glucose uptake of CRC cells. In addition, since the majority of the glucose is secreted as lactate in actively growing cells, lactate production was revealed to decrease as GLS1 was inhibited. Therefore, it was hypothesized that GLS1-mediated glutaminolysis may also induce cell aerobic glycolysis in addition to the induction of glucose uptake via glucose transport. In addition, the synthesis of lactate generates ATP required to maintain intracellular bioenergetics. GLS1 reduction led to a significant reduction of internal ATP levels, thus inhibiting cell growth and progression. Therefore, it was speculated that GLS1 controls the coordination between glutamine and glucose, and restricts cell survival as a metabolic checkpoint.

To evaluate the clinical significance of GLS1 in colorectal cancer, GLS1 expression was examined in 50 pairs of human colorectal tumors and adjacent normal colon samples with IHC analysis. The expression level of GLS1 was upregulated in the colorectal tumor samples, indicating a large scale of CRC exerts aerobic glycolysis and glutamine addiction phenotype. Furthermore, GLS1 expression is negatively associated with differentiation status and positively associated with TNM stage. Thus, the high expression of GLS1 indicated the malignant CRC phenotype in patients, and GLS1 may also dictate the poor differentiation of CRCs. Currently, the GLS1 CB-839 inhibitor is undergoing clinical trials in certain types of cancer, including triple-negative breast cancer and non-small cell lung cancer (13). Consistent with this, in the present study, administration of BPTES (another GLS1 inhibitor) significantly decreased tumor cell growth. Therefore, the present study extends the application of drugs targeting GLS1, indicating the promising role of GLS1 in CRC treatment.

\section{Acknowledgements}

The present study was supported by the National Natural Science Foundation of China (grant no. 81172368), the Wu Jieping Medical Foundation (grant no. 320.6700.1203) and the Science and Technology Innovation Nursery Foundation of Chinese People's Liberation Army General Hospital (grant no. 14KMM30).

\section{References}

1. Siegel R, Desantis C and Jemal A: Colorectal cancer statistics, 2014. CA Cancer J Clin 64: 104-117, 2014.
2. DeBerardinis RJ, Lum JJ, Hatzivassiliou G and Thompson CB: The biology of cancer: Metabolic reprogramming fuels cell growth and proliferation. Cell Metab 7: 11-20, 2008.

3. Dang CV: Links between metabolism and cancer. Genes Dev 26: 877-890, 2012.

4. Vander Heiden MG, Cantley LC and Thompson CB: Understanding the Warburg effect: The metabolic requirements of cell proliferation. Science 324: 1029-1033, 2009.

5. Cairns RA, Harris IS and Mak TW: Regulation of cancer cell metabolism. Nat Rev Cancer 11: 85-95, 2011.

6. Wise DR and Thompson CB: Glutamine addiction: A new therapeutic target in cancer. Trends Biochem Sci 35: 427-433, 2010.

7. Daye D and Wellen KE: Metabolic reprogramming in cancer: Unraveling the role of glutamine in tumorigenesis. Semin Cell Dev Biol 23: 362-369, 2012.

8. Hensley CT, Wasti AT and DeBerardinis RJ: Glutamine and cancer: Cell biology, physiology, and clinical opportunities. J Clin Invest 123: 3678-3684, 2013.

9. Curthoys NP and Watford M: Regulation of glutaminase activity and glutamine metabolism. Annu Rev Nutr 15: 133-159, 1995.

10. Mates JM, Segura JA, Martín-Rufian M, Campos-Sandoval JA, Alonso FJ and Márquez J: Glutaminase isoenzymes as key regulators in metabolic and oxidative stress against cancer. Curr Mol Med 13: 514-534, 2013.

11. Huang F, Zhang Q, Ma H, Lv Q and Zhang T: Expression of glutaminase is upregulated in colorectal cancer and of clinical significance. Int J Clin Exp Pathol 7: 1093-1100, 2014.

12. Pan T, Gao L, Wu G, Shen G, Xie S, Wen H, Yang J, Zhou Y, Tu Z and Qian W: Elevated expression of glutaminase confers glucose utilization via glutaminolysis in prostate cancer. Biochem Biophys Res Commun 456: 452-458, 2015.

13. Gross MI, Demo SD, Dennison JB, Chen L, Chernov-Rogan T, Goyal B, Janes JR, Laidig GJ, Lewis ER, Li J, et al: Antitumor activity of the glutaminase inhibitor CB-839 in triple-negative breast cancer. Mol Cancer Ther 13: 890-901, 2014.

14. Kaadige MR, Elgort MG and Ayer DE: Coordination of glucose and glutamine utilization by an expanded Myc network. Transcription 1: 36-40, 2010

15. Gao P, Tchernyshyov I, Chang TC, Lee YS, Kita K, Ochi T, Zeller KI, De Marzo AM, Van Eyk JE, Mendell JT and Dang CV: $\mathrm{c}-\mathrm{Myc}$ suppression of $\mathrm{miR}-23 \mathrm{a} / \mathrm{b}$ enhances mitochondrial glutaminase expression and glutamine metabolism. Nature 458: 762-765, 2009.

16. Tanaka K, Sasayama T, Irino Y, Takata K, Nagashima H, Satoh N, Kyotani K, Mizowaki T, Imahori T, Ejima Y, et al: Compensatory glutamine metabolism promotes glioblastoma resistance to mTOR inhibitor treatment. J Clin Invest 125: 1591-1602, 2015.

17. Carr EL, Kelman A, Wu GS, Gopaul R, Senkevitch E, Aghvanyan A, Turay AM and Frauwirth KA: Glutamine uptake and metabolism are coordinately regulated by ERK/MAPK during T lymphocyte activation. J Immunol 185: 1037-1044, 2010.

18. Robinson MM, McBryant SJ, Tsukamoto T, Rojas C, Ferraris DV, Hamilton SK, Hansen JC and Curthoys NP: Novel mechanism of inhibition of rat kidney-type glutaminase by bis-2-(5-phenylacetamido-1,2,4-thiadiazol-2-yl)ethyl sulfide (BPTES). Biochem J 406: 407-414, 2007.

19. Stalnecker CA, Ulrich SM, Li Y, Ramachandran S, McBrayer MK, DeBerardinis RJ, Cerione RA and Erickson JW: Mechanism by which a recently discovered allosteric inhibitor blocks glutamine metabolism in transformed cells. Proc Natl Acad Sci USA 112: 394-399, 2015.

20. Williams DM, Schachter J, Drutz DJ and Sumaya CV: Pneumonia due to Chlamydia trachomatis in the immunocompromised (nude) mouse. J Infect Dis 143: 238-241, 1981.

21. Chu D, Zhang Z, Li Y, Wu L, Zhang J, Wang W and Zhang J: Prediction of colorectal cancer relapse and prognosis by tissue mRNA levels of NDRG2. Mol Cancer Ther 10: 47-56, 2011.

22. Kaadige MR, Looper RE, Kamalanaadhan S and Ayer DE: Glutamine-dependent anapleurosis dictates glucose uptake and cell growth by regulating MondoA transcriptional activity. Proc Natl Acad Sci USA 106: 14878-14883, 2009.

23. Tennant DA, Durán RV and Gottlieb E: Targeting metabolic transformation for cancer therapy. Nat Rev Cancer 10: 267-277, 2010.

24. Richard SM and Martinez Marignac VL: Sensitization to oxaliplatin in HCT116 and HT29 cell lines by metformin and ribavirin and differences in response to mitochondrial glutaminase inhibition. J Cancer Res Ther 11: 336-340, 2015. 